\title{
Sensor Network with Unmanned Aerial Vehicle-enabled Wireless Power Transfer: Optimal Clustering and Trajectory Designing
}

\author{
Guiyun Liu, ${ }^{1}$ Wenjun Jiang, ${ }^{1 *}$ Weiqiang Tan, ${ }^{2}$ and Xuejing Lan ${ }^{1}$ \\ ${ }^{1}$ School of Mechanical and Electric Engineering, Guangzhou University, \\ Guangzhou 510006, P. R. China \\ ${ }^{2}$ School of Computer Science and Cyber Engineering, Guangzhou University, \\ Guangzhou 510006, P. R. China
}

(Received July 12, 2019; accepted December 16, 2019)

Keywords: WSNs, WPT, cluster, UAV, path planning, energy consumption

In this work, we investigate the application of an unmanned aerial vehicle (UAV)-enabled wireless power transfer (WPT) system in large-scale wireless sensor networks (WSNs). The specific research described in this paper can be divided into three parts. Firstly, it is well known that the energy consumption of WSNs and the limited-capacity battery of nodes lead to the limited lifetime of WSNs. To improve the lifetime of the WSNs, the UAV's optimal position and the optimal clustering scheme are determined using an improved differential evolution (DE) algorithm in multicluster scenarios. The corresponding energy consumption is determined by comparing different clustering schemes. Meanwhile, the shortest time for the UAV to charge nodes is determined by utilizing the proposed DE algorithm to search for the optimal charging location. Since the UAV usually acts as both a base station and an energy transmitter, the algorithm requires joint optimization of the clustering scheme and the UAV's charging location. Secondly, as the scale of WSNs increases, some areas will be in the "dead" state within the WSNs, which will greatly affect the performance. On the basis of the above studies, an improved local permutation algorithm is applied to plan an optimal path subject to the dormancy rate constraint in all regions. With the algorithm, the UAV needs to "rescue" the target region and charge regions along the path as much as possible. Finally, numerical results show that the optimized UAV trajectory can significantly improve the energy transmission efficiency, and the proposed optimal path planning improves the lifetime of WSNs.

\section{Introduction}

Wireless power transfer (WPT) is widely considered a key technology in our time. ${ }^{(1)}$ What makes this technology particularly attractive is the nonrequirement of line of sight (LOS) and its sensitivity to the neighboring environment. There are many principles of WPT, such as magnetic resonance, magnetic coupling, and capacitive coupling. ${ }^{(2-4)}$ Owing to the maturity of magnetic resonance coupling, it has been quickly applied to the commercial market. ${ }^{(5)}$

In recent years, renewable sensor networks (RSNs) with WPT have been regarded as the next generation of wireless sensor networks (WSNs). ${ }^{(6,7)}$ Through periodic WPT, WSNs can have 
a virtually infinite lifetime and remain operational forever. ${ }^{(8)}$ The optimization problem is to maximize the ratio of the wireless charging vehicle's rest time to the cycle time I, as described in Ref. 8. Many groups have studied a mobile robot's motion pattern using a varying-parameter convergent differential neural network. ${ }^{(9-12)}$ Different from the objective and conditions described in Ref. 8, we propose a new idea of employing a new unmanned aerial vehicle (UAV)-enabled WPT system to charge a WSN periodically. The optimal strategy of the charge and path plan for the UAV can ensure that sensors have sufficient energy and performance improvements. However, because of the severe propagation loss of RF signals over distance, the efficiency of WPT becomes very low. As a result, to provide a wireless energy supply to energy receivers (ERs) or sensors, some approaches have been proposed to solve these problems. In Refs. 13-17, different approaches to multiantenna beamforming and waveform optimization were proposed to improve the efficiency of wireless energy transmission from different aspects.

As the node density increases in WSNs, a multinode charging technology is applied to accomplish energy transmission to many sensors simultaneously. The technology described in Ref. 18 is for simply charging one node at a time and is not applicable to WSNs. Specifically, Kurs et al. developed a multinode charging technology by appropriately tuning coupled resonators. ${ }^{(19)}$ It was proved that the total output efficiency of charging multiple nodes is better than that of charging each node individually.

Different from previous studies, we applied a multinode charging technology to perform energy transmission from a UAV to sensors. The determination of the optimal position for efficient energy transmission of the UAV is the first step in this work. Considering largescale sensor networks, a path plan for the UAV is proposed to improve the energy transmission efficiency of the entire network. In detail, the UAV is considered to charge nodes in a region, as shown in Fig. 1. Successful charging of multiple nodes simultaneously by a UAV has been reported. ${ }^{(20,21)}$ As shown in Fig. 1, there are different clusters in a region. Cena et al. used the adaptive cluster-head round-robin scheme to optimize the energy consumption of communication among nodes in each cluster. ${ }^{(22)}$ Different from Ref. 23, different clusters in a region lead to various energy consumption situations. Therefore, the way of choosing clustering

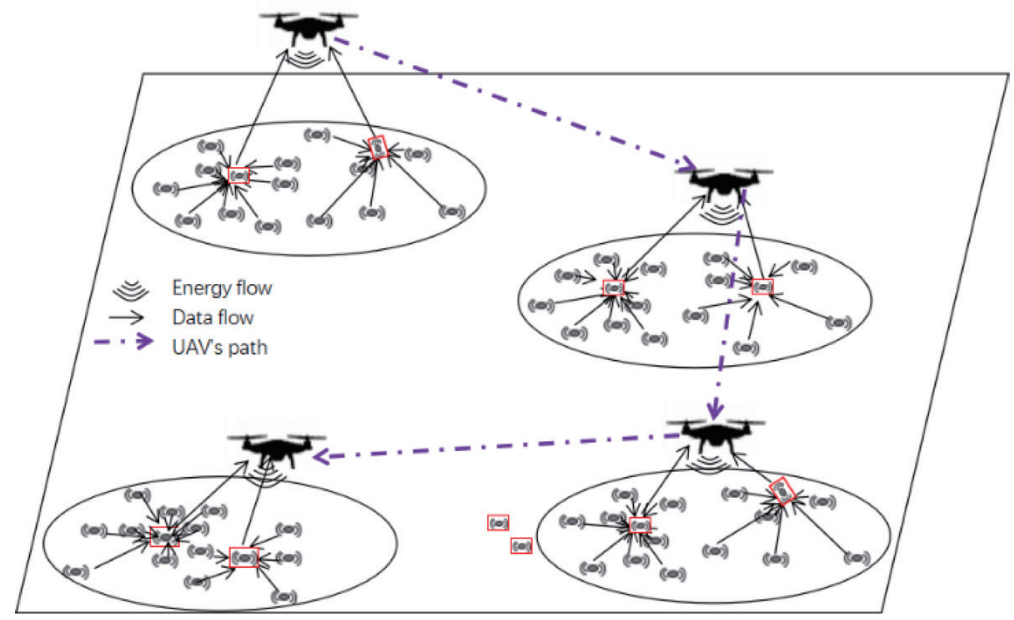

Fig. 1. (Color online) An example of a UAV-enabled WSN. 
schemes and optimal positions for the UAV becomes an issue. For simplification, every node can be considered a cluster head regardless of the residual energy of the nodes and the cluster head rotation. Through a differential evolution (DE) algorithm, the optimal position for the UAV to charge nodes can be obtained. Motion planning ${ }^{(24)}$ and a cooperative multiagent search with resource constraints ${ }^{(25)}$ have also been considered.

In this study, large-scale WSNs are segmented into several regions, as mentioned above. To charge sensors in one region, the UAV only needs to visit the optimal position of the region. ${ }^{(26)}$ On the basis of the conventional energy charging model, a method of optimizing the traveling path is proposed. This optimization problem is constrained by the dormancy rate in all the regions and the requirement of the shortest path. By employing the local permutation algorithm, an optimal path for the UAV is efficiently determined. According to our numerical results, this solution enables us to address the charging problem in WSNs.

This paper is organized as follows. In Sect. 2, the mathematical model in this study is described. In Sect. 3, we present two formulations of two optimization problems, and the optimal solutions for the two problems are developed in Sects. 3.1 and 3.2. In Sect. 4, numerical results are presented to demonstrate the validity of the solutions. In Sect. 5, we conclude this paper.

\section{Mathematical Modeling}

In this section, the energy charging behavior and energy consumption model of sensors are described in detail.

\subsection{Energy charging behavior}

The wireless channel between the UAV and each ER is normally LOS-dependent, as described in Refs. 24 and 27. The UAV hovers at position $(x(t), y(t), H)$ and broadcasts wireless energy to charge the sensors. $I$ denotes the set of all nodes. Each sensor $i_{g} \in I$ is at a fixed location on the ground, denoted by $(x(t), y(t))$. The channel power gains from the UAV to the sensor $i_{g}\left(i_{g} \in I\right)$ are modeled as

$$
h_{k}(t)=\frac{\beta_{0} \times \eta}{\left(x(t)-x_{k}\right)^{2}+\left(y(t)-y_{k}\right)^{2}+H^{2}},
$$

where $\beta_{0}$ denotes the channel power gained at a reference distance $d_{0}$ of $1 \mathrm{~m}$. The power harvested by the sensor at time $t$ is thus given by

$$
Q_{i}(x(t), y(t))=h_{k}(t) \times P=\frac{\beta_{0} \times \eta \times P}{\left(x(t)-x_{k}\right)^{2}+\left(y(t)-y_{k}\right)^{2}+H^{2}},
$$

where $P$ is a constant transmitted power of the UAV and $\eta$ denotes the energy conversion efficiency of the rectifier at each sensor. 


\subsection{Energy consumption of sensors}

The model for the radio sensor energy dissipation is described in this section. When transmitting an $l$-bit message at distance $d$, the energy consumption can be given by

$$
E_{T X}(l, d)= \begin{cases}l \times E_{\text {elec }}+l \times E_{D A}+l \times \varepsilon_{f s} \times d^{2} & d<d_{0} \\ l \times E_{\text {elec }}+l \times E_{D A}+l \times \varepsilon_{m p} \times d^{2} & d>d_{0} .\end{cases}
$$

When receiving a message, the energy consumption of the radio can be expressed as

$$
E_{R X}(l, d)=l \times E_{\text {elec }},
$$

where $\varepsilon_{f s}$ and $\varepsilon_{m p}$ are both energy factors, and $E_{\text {elec }}, E_{D A}$, and $d_{0}$ are the electronics energy, the energy consumption for data fusion, and a distance threshold, ${ }^{(28)}$ respectively. There are many factors, such as modulation, coding, filtering, and spreading of signals, included in the electronics energy $E_{\text {elec }} . E_{D A}$ indicates the unit fusion energy consumption. The energy parameters are set as $E_{\text {elec }}=100 \mathrm{~nJ} / \mathrm{bit}, \varepsilon_{f s}=10 \mathrm{pJ} / \mathrm{bit} / \mathrm{m}^{2}$, and $\varepsilon_{f_{s}}=0.0013 \mathrm{pJ} / \mathrm{bit} / \mathrm{m}^{4}$. ${ }^{(29,30)}$

\section{Problem Formulation and Statement}

As shown in Fig. 2, the WSN is divided into several regions labeled (1), (2), (3), and so on. The node identified by the triangle is the cluster head in the region, and the other nodes are ordinary nodes. I represents the set of nodes. $G=\left\{g \mid g=1,2,3, \ldots, G^{\prime}\right\}$ denotes the set of regions. $I_{g}=\left\{i_{g} \mid i_{g}=1_{g}, 2_{g}, 3_{g}, \ldots,\right\}$ shows the node set of the $g$ th region.

Below is the relationship between the set of nodes and the regions.

$$
I=\bigcup I_{g}
$$

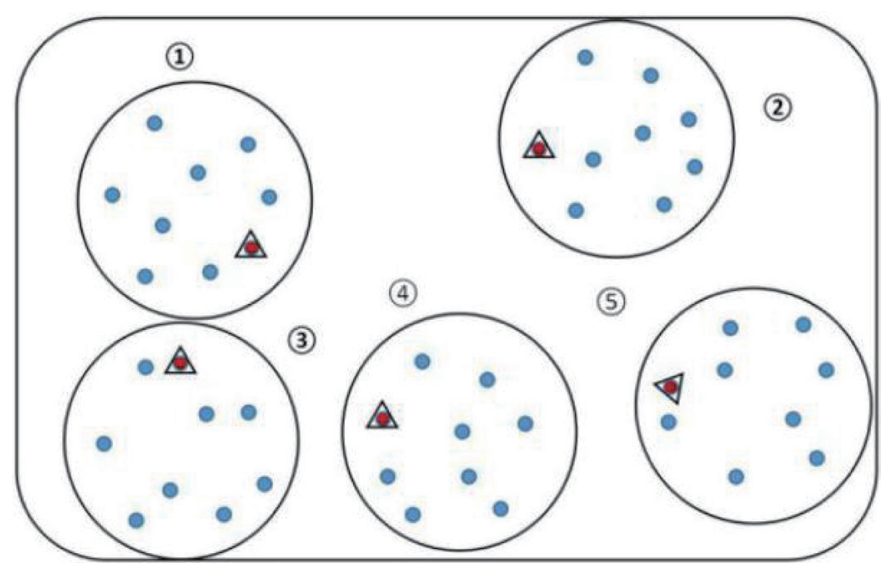

Fig. 2. (Color online) Illustration of WSNs. 
The total number of clustering schemes is $M=N_{1} \times N_{2} \times \ldots \times N_{\mathrm{g}} \times \ldots \times N_{G^{\prime}}$, and $N_{g}$ represents the number of clusters in the $g$ th region. It is assumed that sensors generate or gather $l$-bit data before being charged. $e_{i}=E_{T X}\left(l, d_{i, i_{j}}\right)$ is the energy consumption of node $i . e_{i_{j}}=E_{T X}\left(l, d_{i_{j}, B}\right)$ is the energy consumption of cluster head $i_{j}$. To achieve this optimization goal, the following tasks must be accomplished. From the mathematical modeling that we discussed in Sect. 2, one performance objective is to minimize $\tau$, which is the time required for the UAV to charge the sensors. Considering the above scenario, the optimization problem can be formulated as follows:

$$
\begin{gathered}
P 1-a: \min \tau \\
\text { s.t. } x_{\text {min }} \leq x \leq x_{\text {max }} \\
y_{\text {min }} \leq y \leq y_{\text {max }} \\
t_{i}^{m}=\frac{e_{i}^{m}}{Q_{i}^{m}(x, y)}, \\
t_{i_{j}}^{m}=\frac{e_{i_{j}}^{m}}{Q_{i_{j}}^{m}(x, y)} .
\end{gathered}
$$

Here, $y_{\min } \leq y \leq y_{\max }$ and $x_{\min } \leq x \leq x_{\max }$ indicate the WSN range in which the UAV charges nodes. $e_{i}^{m}$ and $e_{i_{j}}^{m}$ are the energy consumptions of the nodes and cluster head in the $m$ th scheme, respectively. $t_{i}^{m}$ and $t_{i_{j}}^{m}$ are the times required for the nodes and cluster head to be fully charged in the $m$ th scheme, respectively. $Q_{m}^{i}(x, y)$ and $Q_{m}^{i_{j}}(x, y)$ are the power provided by the UAV to charge the WSN in the $m$ th scheme.

In this study, the nodes are fully charged to $E_{\max }$. As shown in Fig. 3, some nodes may experience saturation before all the nodes are fully charged. Therefore, the longest time

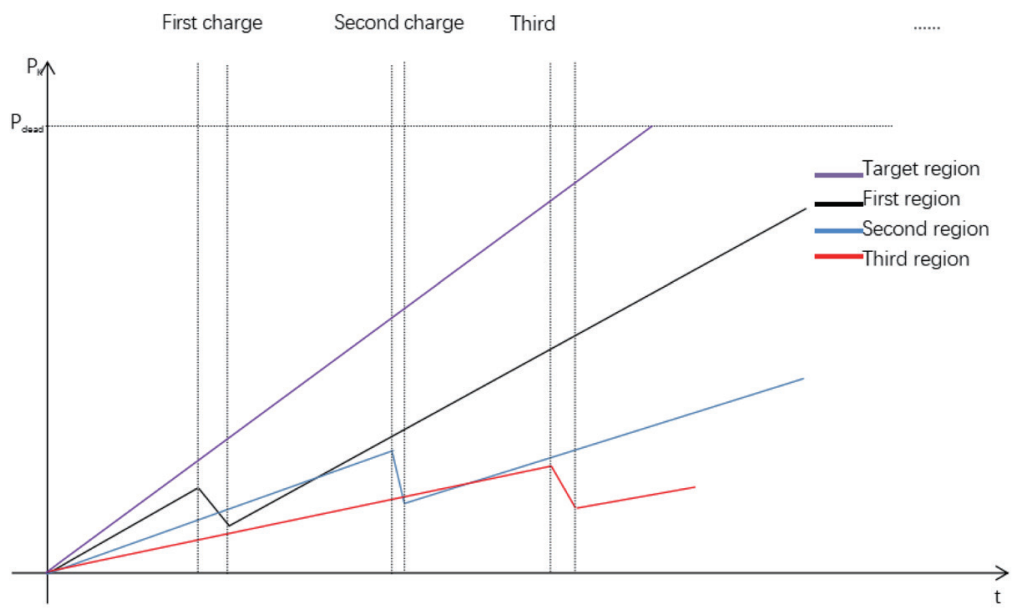

Fig. 3. (Color online) States of nodes. 
$t_{\max }=\max \left(t_{i}\right)$ can be regarded as the charging time. Furthermore, there are different energy consumption situations owing to different clusterings. On the basis of the analysis above, the optimal problem can be reformulated as follows.

$$
\begin{gathered}
P 1-b: \max _{x, y} \min E_{i_{g}}^{m}=\left\{e_{i_{g}}^{m} \mid i_{g} \in I_{g}, 1 \leq m \leq M\right\} \\
\text { s.t. } \quad(7),(8),(9), \text { and (10) }
\end{gathered}
$$

Let $x^{*}$ and $y^{*}$ denote the coordinates of an optimal UAV location that maximizes the minimum of the function $E_{i_{g}}^{m}$, i.e.,

$$
\left(x^{*}, y^{*}\right)=\arg \max _{x, y} \min E_{i_{g}}^{m} .
$$

The improved DE algorithm is applied to solve the optimization problem.

From the above scenarios, there exists an optimal and practical strategy for the UAV to charge sensors. An optimal path plan can be proposed for the UAV to charge sensors. It is meaningful to shorten the total time $\tau$ taken for the UAV to charge the sensor network. Prolonging the lifetime of the network is also an important target. In large-scale WSNs, a high dormancy rate greatly affects the performance. Therefore, it is necessary for the UAV to have an optimal path plan.

For example, Fig. 3 shows the dormancy rate level of the region. When the UAV flies to the target region, it should "rescue" the target region as soon as possible, i.e., ensure that all nodes have sufficient power by charging as necessary. If not, the target region will be close to the "paralyzed" state, which will affect the performance of the whole WSN. In Sect. 3.2.2, $T_{\text {need }}$ represents the longest time for which the UAV flies to "rescue" the target region as soon as possible.

In practical applications, the UAV is required to do more things besides saving the target region. Within the allowable time, the UAV is required to rescue as many regions as possible. The problem can be formulated as follows.

$$
\begin{gathered}
P 2: \max _{x, y} \bar{\rho} \\
\text { s.t. } \quad T=\sum_{l=0}^{L} t_{i}+\sum_{i=0}^{I} t_{i}<T_{\text {need }}
\end{gathered}
$$

In the above, $\bar{\rho}$ is the average dormancy rate. $\sum_{l=1}^{L} t_{l}$ and $\sum_{i=1}^{l} t_{i}$ are the flight time of the UAV and charging time, respectively. The improved local permutation algorithm is applied to solve P2. More details about the path plan are described in Sect. 3.2. 


\subsection{DE algorithm for cluster head election and optimal UAV position}

The WSN is composed of numerous tiny nodes that have limited battery power. Through sensing or controlling the physical parameters, the nodes can interact with their environment. These nodes collect data and carry out data fusion. As shown in Fig. 4, the sensors transmit their information to the cluster head through a single hop. Firstly, the cluster head integrates the received information into data packets. Then, the information is transmitted to the mobile base station UAV through a single hop.

Clustering plays an important role in improving the network scalability and lifetime. The nodes transmit their data to their cluster heads. There are a number of clustering algorithms, such as LEACH, particle swarm optimization, genetic algorithms, and DE. In this study, we propose a clustering algorithm based on the DE algorithm. In particular, this algorithm can determine the optimal position for the UAV, which is the mobile base station and the energy provider. Different schemes of cluster head selection correspond to different charging times and different optimal positions for the UAV. As shown in Fig. 5, nodes C, D, E, and F are selected

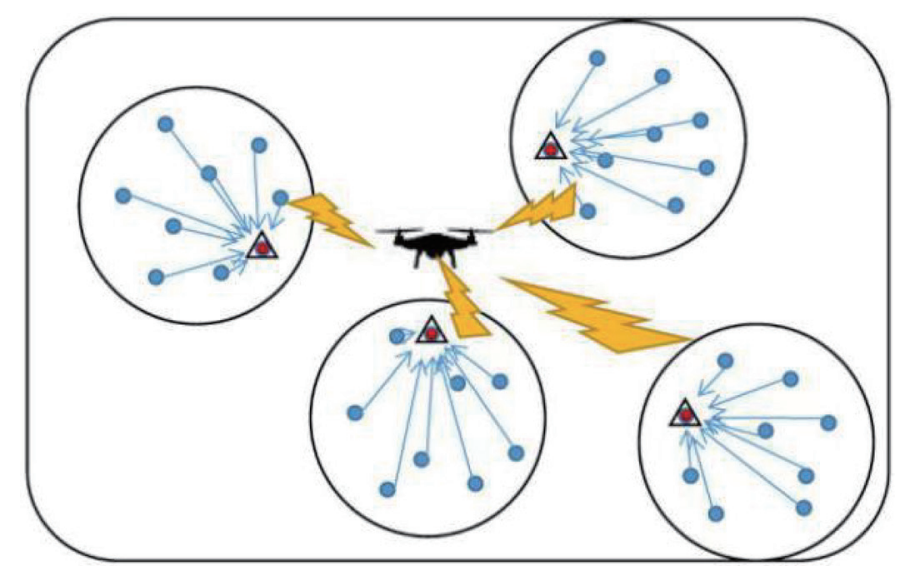

Fig. 4. (Color online) UAV-enabled WSN.

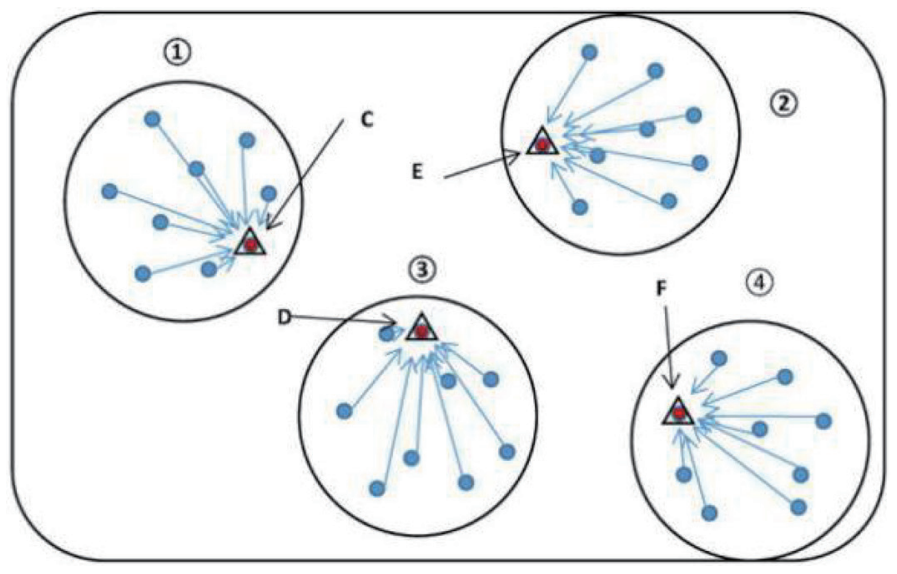

Fig. 5. (Color online) Cluster head selection. 
as cluster heads by the DE algorithm. Regions labeled (1), (2), and (3) correspond to the 1st region, the 2 nd region, and the 3 rd region, respectively. The UAV hovers at the optimal position where it spends the shortest time to charge nodes.

\subsubsection{Detailed explanation of improved optimization algorithm}

The pseudocode for the improved DE algorithm proposed in this study is shown as Algorithm 1.

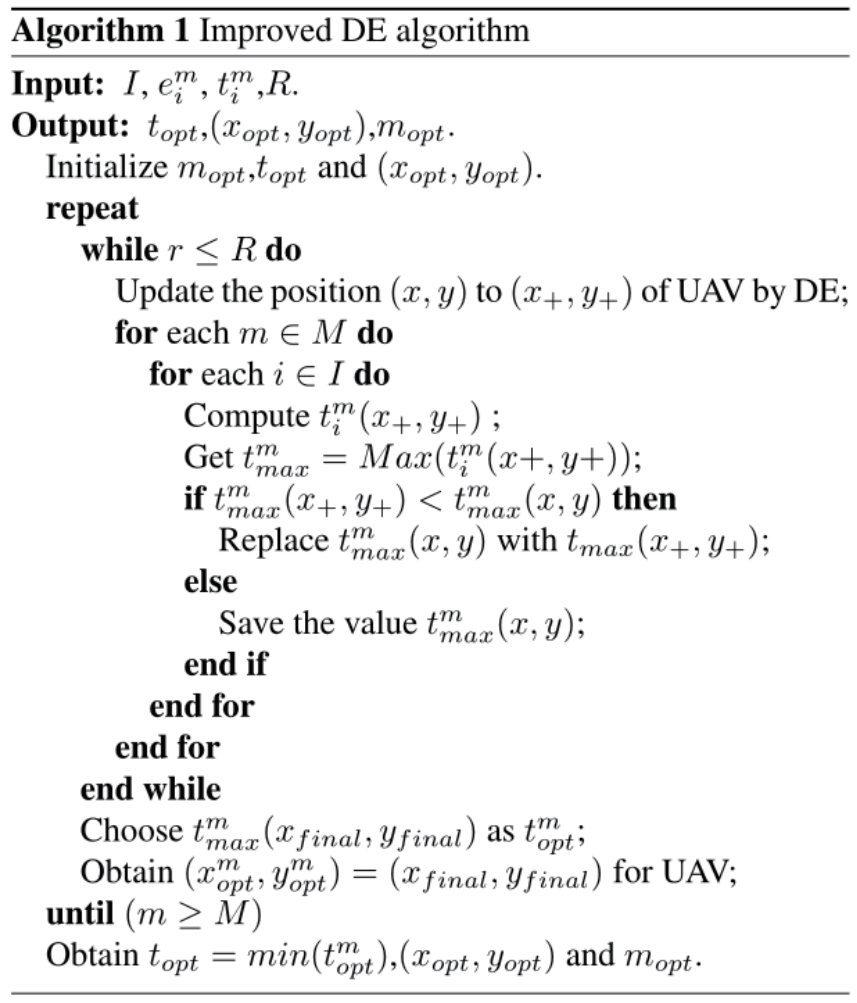

In this algorithm, $m$ is the $m$ th clustering scheme and $M$ is the total number of schemes. $i$ denotes one node in the region. In the $m$ th scheme, $e_{i}^{m}$ and $t_{i}^{m}$ represent the energy and the charging time, respectively, of the $i$ th node, including cluster head $i_{j}$. $R$ represents the number of iterations for the DE algorithm. $\left(x_{+}, y_{+}\right)$is the updated position of the UAV, which is based on the former position $(x, y)$. When the UAV hovers at $\left(x_{+}, y_{+}\right)$and charges nodes, $t_{i}^{m}\left(x_{+}, y_{+}\right)$ denotes the charging time of node $i$ in the $m$ th scheme. In the $m$ th scheme, $t_{\max }^{m}$ is the time required for the UAV to charge nodes in all the regions. When the UAV hovers at the final position ( $\left.x_{\text {final }}, y_{\text {final }}\right)$ updated by the algorithm, $t_{\text {max }}^{m}\left(x_{\text {final }}, y_{\text {final }}\right)$ represents the time required for the UAV to charge nodes in the $m$ th scheme. $t_{o p t}^{m}$ is the optimal required time for the UAV to fully charge all the nodes in the $m$ th scheme, and $\left(x_{o p t}^{m}, y_{o p t}^{m}\right)$ is the corresponding position for the UAV. $t_{\text {opt }}$ is the optimal required time for the UAV to fully charge all the nodes among all the schemes $M$. $\left(x_{o p t}^{m}, y_{\text {opt }}^{m}\right)$ represents the optimal position at which the UAV should hover under the optimal scheme $m_{o p t}$, which is $\left(x^{*}, y^{*}\right)$ in Sect. 3. $i_{j}$ denotes the cluster head. The key principles of this algorithm can be represented by the flowchart shown in Fig. 6. 


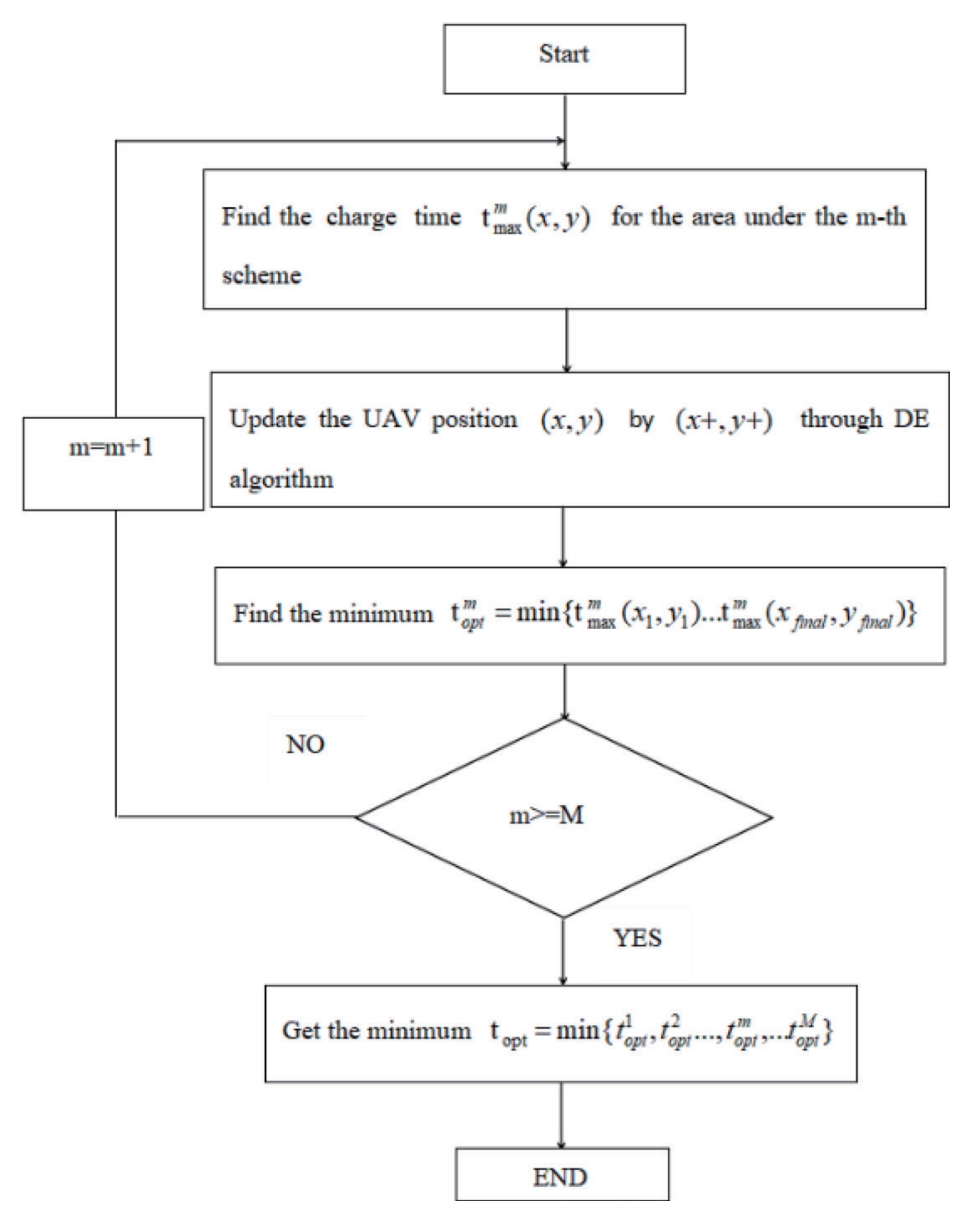

Fig. 6. Flowchart of the improved DE algorithm.

\subsection{Path planning for the UAV}

In practical applications, it is meaningful to shorten the total time needed for the UAV to charge sensors. Prolonging the lifetime of the WSN is also an important target. However, it is very difficult to find the shortest time necessary for the UAV to charge nodes, just as P2 shows. For simplification, the optimal position $\left(x_{o p t}, y_{\text {opt }}\right)$ for the UAV to charge nodes in a region is regarded as path point $o$ here. $o^{\prime}$ is a point in the neighborhood of $o$. As shown in Fig. 7 , the points connected by dotted lines are the points around path point $o$. For example, $o_{1}{ }^{\prime}$ and $o_{2}{ }^{\prime}$ belong to the set o.c $\left(o_{1}{ }^{\prime}, o_{2}{ }^{\prime} \in O . c\right) . c$ is the category of nodes between $S$ and $T$, and q.c represents all the categories of nodes (o.c $\in$ q.c) .

In this study, a local permutation algorithm and its improved algorithm are used to plan the path and prolong the lifetime of the network. A recursive algorithm is applied to find the shortest path, and an iterative method is used for optimization. The basic principles of the algorithms are described as follows. Firstly, choose the target points $T$ in the flight range through perceptual behavior, and plan an initial shortest path from $S$, which can be shown as the result sequence $\sigma$. Secondly, during each step of the iteration, choose the path point $o$ with 


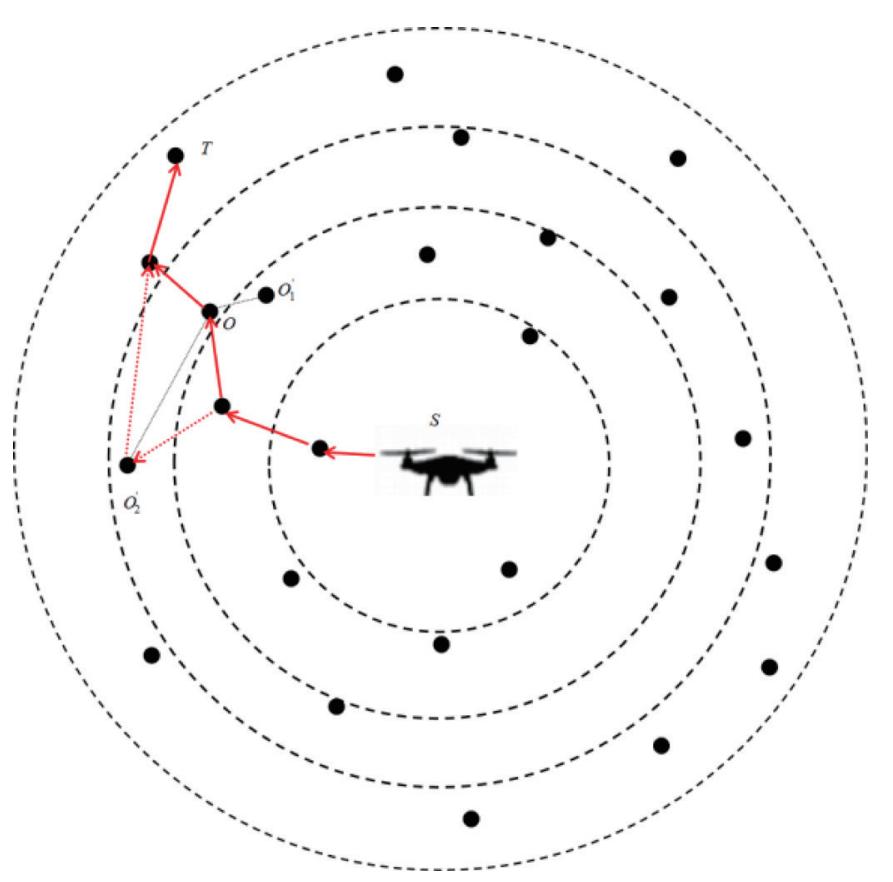

Fig. 7. (Color online) Illustration of flight plan for the UAV.

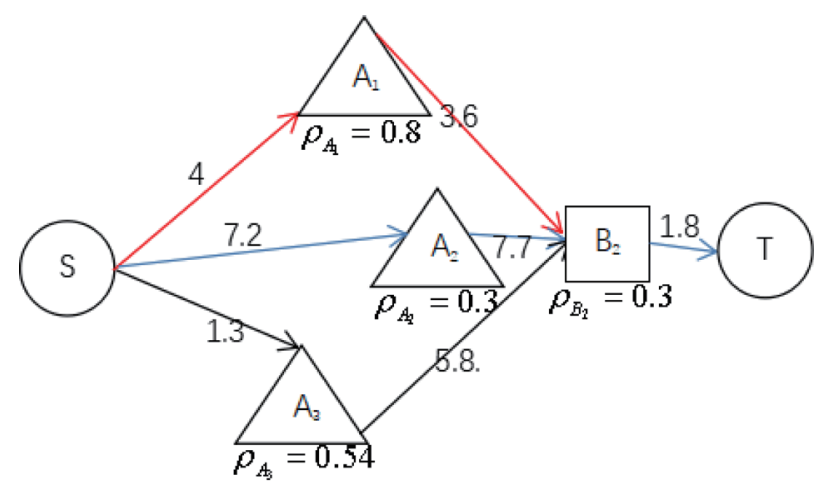

Fig. 8. (Color online) Index process of the local permutation algorithm.

the lowest dormancy rate o.p in path $\sigma$. Thirdly, replace $o$ with $o^{\prime}$ where $o^{\prime} \in$ o.c. For example, as shown in Fig. 8, $A_{2}$ can be replaced by $A_{1}$ or $A_{3}$, which both belong to the set $A$.c $=\left\{A_{1}, A_{3}\right\}$. Fourthly, test all the possible points in set $o . c$ that satisfy $t<t_{\text {need }}$ or others.

To explain the algorithm, the local permutation algorithm and its improved algorithm to solve $\mathrm{P} 2$ are shown in detail below.

\subsubsection{Local permutation algorithm}

The index process of the local permutation algorithm is given in detail in Fig. 8. Firstly, the shortest path $S \rightarrow A_{2} \rightarrow B_{2} \rightarrow T$ is found from $S$ to $T$, and the average dormancy rate along this 
path is $\bar{\rho} . \rho_{0}\left(\rho_{0}<1\right)$ is the target dormancy rate, which can be set in accordance with the actual situation. The numbers next to the arrows stand for distances between points. If the paths do not meet,

$$
\bar{\rho}<\rho_{0},
$$

they will be updated. Assuming that the dormancy rate at point $A_{2}$ is the lowest along the path, it will be replaced by another point whose dormancy rate is higher. As Fig. 8 shows, $A_{2}$ can be replaced by $A_{1}$ or $A_{3}$ to construct a new path. Finally, a new path is updated with $A_{2}$ replaced by $A_{1}$ because the distance change is smaller than the change in $A_{3} . S \rightarrow A_{1} \rightarrow B_{2} \rightarrow T$ is the new path, and the current average dormancy rate is $\bar{\rho}$.

The pseudocode of the local permutation algorithm is given as Algorithm 2.

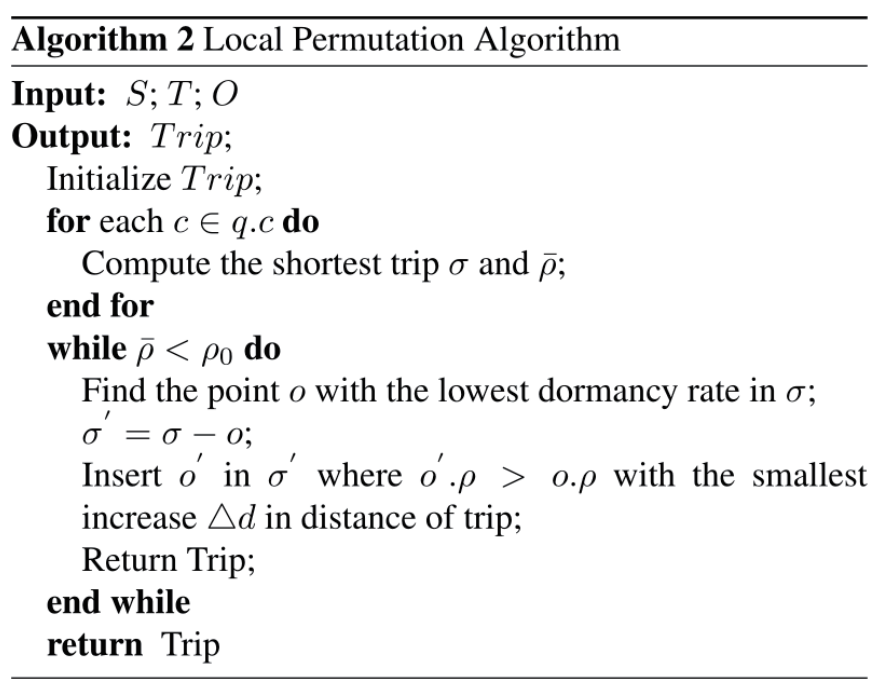

\subsubsection{Improved local permutation algorithm}

To plan a more suitable path for $\mathrm{P} 2$, the improved local permutation algorithm is introduced here. Unlike the local permutation algorithm, there is no requirement of $\bar{\rho}<\rho_{0}$ in the improved local permutation algorithm. The numbers next to the arrows stand for distances $d_{l}$ between points. $V$ represents the speed of the UAV between path points $o$. The basic principle of the improved local permutation algorithm is as follows.

Firstly, find the shortest path (the initial path) $S \rightarrow A_{2} \rightarrow B_{2} \rightarrow C_{2} \rightarrow T$. The average dormancy rate along this path is $\bar{\rho}$. Secondly, find the point (such as $A_{2}$ shown in Fig. 9) that has the lowest dormancy rate along the initial path. Thirdly, find the point with a higher dormancy rate around $A_{2}$; there are two points $\left(A_{1}\right.$ and $\left.A_{3}\right)$ that can replace $A_{2}$. The path constructed with $A_{2}$ replaced by $A_{1}$ is longer than that constructed with $A_{2}$ replaced by $A_{3}$. Through $k$ iterations, the path is gradually updated. Therefore, the new path $S \rightarrow A_{3} \rightarrow B_{2} \rightarrow C_{2} \rightarrow T$ is updated from the initial path, and the current average dormancy rate is $\bar{\rho}_{\text {new }}$. Finally, the time must be checked to determine whether it meets the following requirement shown as $\mathrm{P} 2$. 


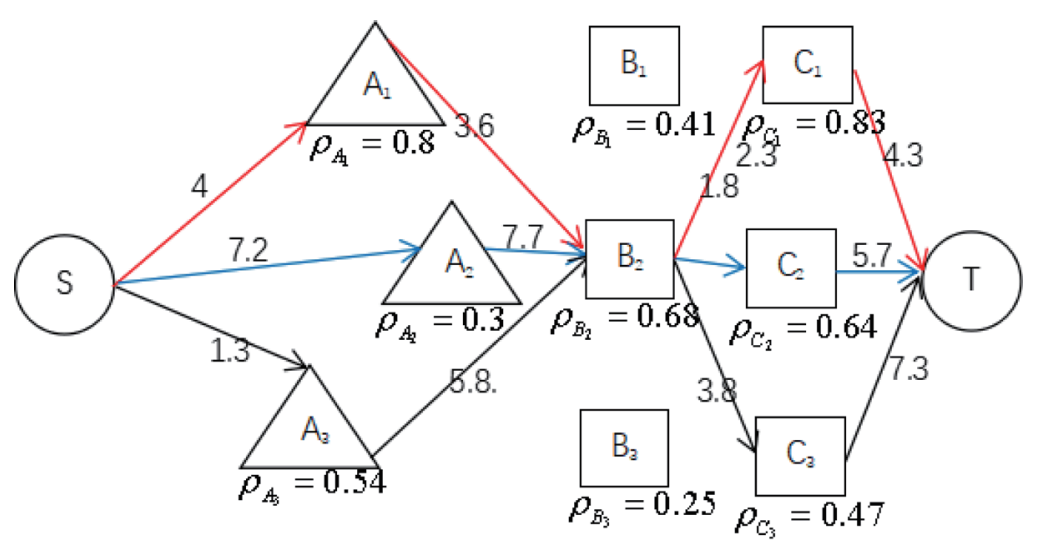

Fig. 9. (Color online) Diagram of the improved local permutation algorithm.

$$
t_{\text {path }}=\sum_{l=1}^{L} \frac{d_{l}}{V}+\sum_{i=1}^{I} t_{i}<T_{\text {need }}
$$

Here, $T_{\text {need }}$ is the maximum time taken for the UAV to arrive at the target point $T$, and the UAV can spend more time hovering at path points if $t_{\text {path }}<T_{\text {need }}$. As shown in Fig. 9, the black line $\left(S \rightarrow A_{3} \rightarrow B_{2} \rightarrow C_{3} \rightarrow T\right)$ represents the final path updated from the initial path (the red line; $\left.S \rightarrow A_{1} \rightarrow B_{2} \rightarrow C_{1} \rightarrow T\right)$.

The diagram of the algorithm is shown in Fig. 9, and the pseudocode for the algorithm is given as Algorithm 3.

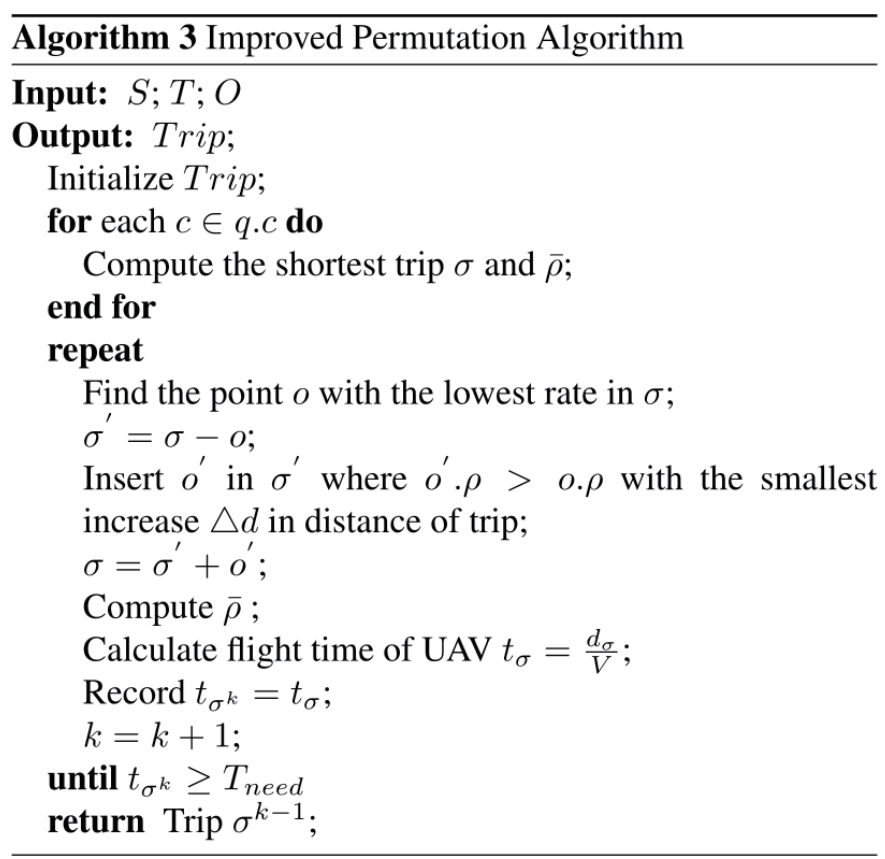




\section{Simulation and Analysis}

In this section, some numerical results are presented to demonstrate how to obtain the optimal scheme for the UAV and WSNs. The optimal path plan for the UAV to perform energy transfer is proposed.

\subsection{Simulation settings}

The following experiments are conducted under the MATLAB environment. Sensor nodes are assumed to be deployed over a $1000 \times 1000 \mathrm{~m}^{2}$ square area. The base station is at $(500,500)$ (in $\mathrm{m})$. The WCV's home service station is assumed to be at the origin. The speed of the WCV is $V=5 \mathrm{~m} / \mathrm{s}$. Let $E_{\max }=10.8 \mathrm{~kJ}$ and $E_{\min }=540 \mathrm{~J}$.

\subsection{Results of P1 and P2}

For simplification, one result in P1 is shown for a small network. As shown in Fig. 10(a), the position represented by the small red circle is the shortest time required for the UAV to charge nodes. At the same time, the scheme in Fig. 10(a) is the best among all the possible schemes for clustering. Figure 10(b) shows the graph of the object function value, showing its iterative convergence.

In Sect. 3.2.2, the optimal path is found by the improved local permutation algorithm. In Fig. 7, path planning is based on optimal positions for the UAV in several regions, as described in Sect. 3.1.

Figure 11 shows the path plan obtained through the local permutation algorithm discussed in Sect. 3. However, the path plan in Sect. 3.2.1 needs to meet the target dormancy rate $\rho_{0}$. Because the target dormancy rate $\rho_{0}$ is a constant, the optimization of the dormancy rate cannot be considered in this path plan.

Below are some simulations of the improved permutation algorithm described in Sect. 3.2.2. As shown in Fig. 12(a), if $T_{\text {need }}=9$, there is one optimal path for P2.

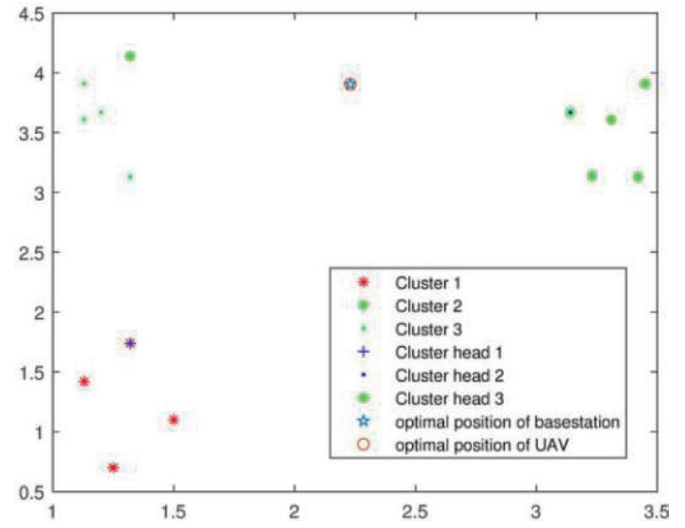

(a)

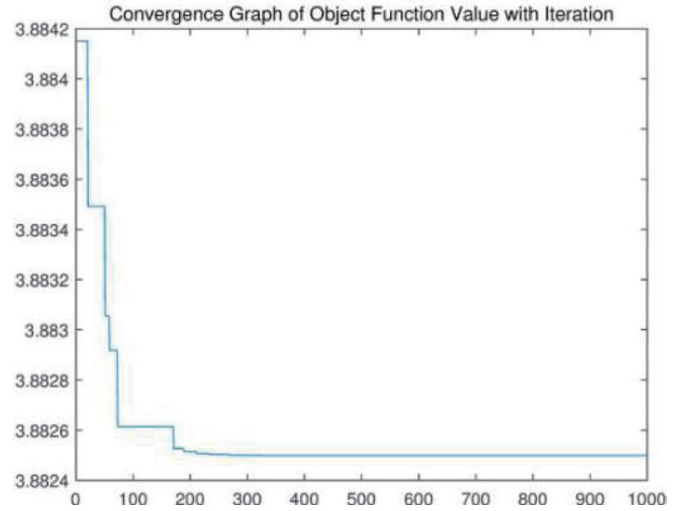

(b)

Fig. 10. (Color online) (a) Solution for P1-b and (b) convergence graph of the object function value with the improved DE algorithm. 


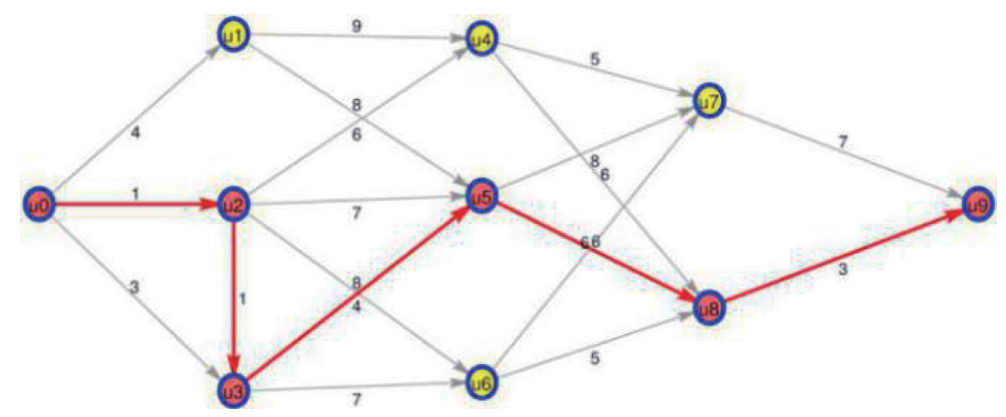

Fig. 11. (Color online) Path plan in Subsect. 3.2.1.

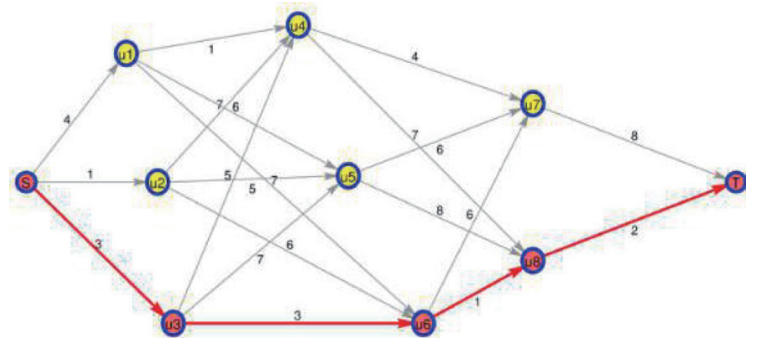

(a)

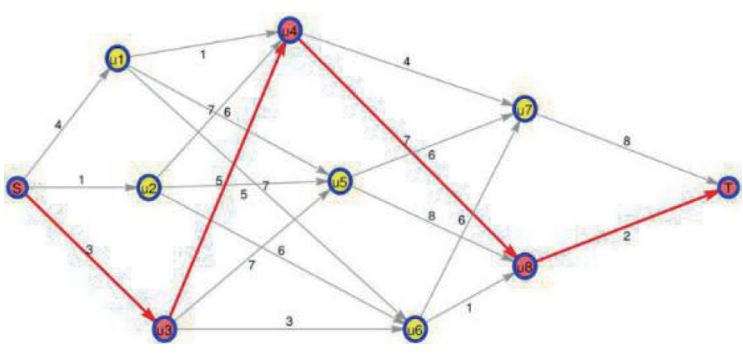

(b)

Fig. 12. (Color online) Corresponding path plan (a) when $T_{\text {need }}$ is set to 9 and 11 or (b) when $T_{\text {need }}$ is set to 19 .

When $T_{\text {need }}=11$, the corresponding path plan is also shown in Fig. 12(a). There are no optimal paths that meet $t_{\text {path }}=9<T_{\text {need }}=11$ or have a lower dormancy rate $\bar{\rho}$.

When $T_{\text {need }}=19$, the corresponding path plan is shown in Fig. 12(b). The UAV flies to the charge target region along the path where the UAV can rescue more regions in the shortest time. The time of the flight along this path is $t_{\text {path }}=17$, which meets the following requirement in $\mathrm{P} 2$.

$$
t_{\text {path }}=\sum_{l=1}^{L} t_{l}+\sum_{i=1}^{I} t_{i}<T_{\text {need }}
$$

For the ease of understanding, dormancy rates are compared among possible paths when $T_{\text {need }}$ is set to 11 or 19 . From Fig. 13(a), the average dormancy rate with $t_{\text {path }}=9$ is smaller than that with $t_{\text {path }}=10$ when $T_{\text {need }}$ is set to 11 or 19 . Clearly, the path with $t_{\text {path }}=9$ is better than other possible paths. Similarily, the path with $t_{\text {path }}=10$ has a higher average dormancy rate than the others, which means that the UAV can spend more time hovering at path points to rescue them (charge them) before the UAV arrives at the target point $S$. The higher average dormancy rate of the path means that these path points need to be rescued more than the others. 


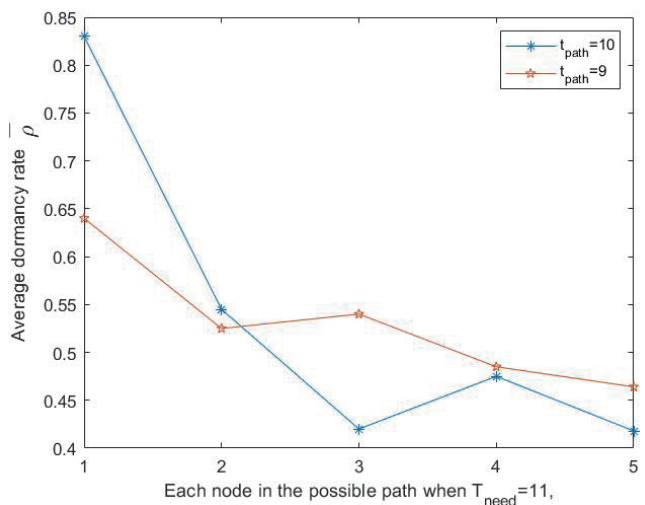

(a)

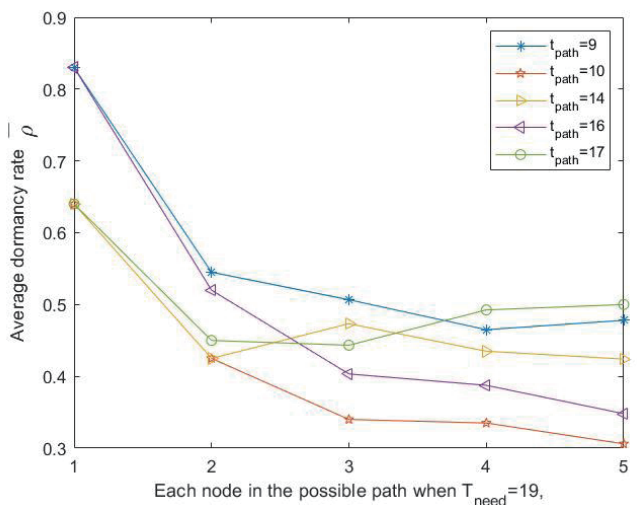

(b)

Fig. 13. (Color online) Comparison of dormancy rates among possible paths when (a) $T_{\text {need }}$ is set to 11 and (b) $T_{\text {need }}$ is set to 19 .

\section{Conclusions}

In this study, a UAV-enabled WPT system was applied in a large-scale WSN. First, an improved DE algorithm was developed by jointly optimizing the clustering schemes and the positions for the UAV to charge nodes. Second, with the improved local permutation algorithm, the optimal path plan for the UAV to visit the regions and charge nodes successively was determined. Finally, using the numerical results, we demonstrated a UAV-enabled WPT system while addressing the lifetime problem for large-scale WSNs.

\section{Acknowledgments}

This work was supported by the National Natural Science Foundation of China (61403089, 61803111, and 51575115) and the Postgraduate Innovative Capability Cultivation Program of Guangzhou University under Grant No. 2018GDJC-M21.

\section{References}

1 O. Jonah and S. V. Georgakopoulos: IEEE Trans. Antennas Propagat. 61 (2012) 1378. https://doi.org/10.1109/ TAP.2012.2227924

2 S. Bi, C. K. Ho, and R. Zhang: IEEE Trans. Antennas Propagat. 53 (2015) 117. https://doi.org/10.1109/ MCOM.2015.7081084

3 H. Dai, Y. Huang, C. Li, S. Li, and L. Yang: IEEE Commun. 11 (2017) 326. https://doi.org/10.1049/ietcom. 2015.1192

4 A. K. RamRakhyani, S. Mirabbasi, and M. Chiao: IEEE Trans. Circuits Syst. 5 (2011) 48. https://doi. org/10.1109/TBCAS.2010.2072782

5 F. Zhang, X. Liu, S. A. Hackworth, R. J. Sclabassi, and M. Sun: IEEE. Syst. Appl. Workshop 34 (2009) 84. https://doi.org/10.1109/LISSA.2009.4906715

6 A. Flammini and E. Sisinni: IEEE. Syst. Appl. Workshop 87 (2014) 672. https://doi.org/10.1109/ LISSA.2009.4906715

7 L. Jie, Y. Gao, and H. Wang: Telecommun. Syst. 10 (2019) 541. https://link.springer.com/article/10.1007/ s11235-018-00541-9 
8 Y. Shi, L. Xie, Y. T. Hou, and H. D. Sherali: Proc. IEEE INFOCOM 5 (2011) 1350. https://doi.org/10.1109/ INFCOM.2011.5934919

9 Y. Hu, H. Su, and L. Zhang: Robotics 8 (2019) 64. https://doi.org/10.3390/robotics8030064

10 Y. Hu, X. Wu, and P. Geng: IEEE Trans. Ind. Electron. 66 (2018) 7788. https://doi.org/10.1109/ TIE.2018.2884240

11 R. Cui, L. Chen, C. Yang, and M. Chen: IEEE Trans. Ind. Electron. 64 (2017) 6785. https://doi: 10.1109/ TIE.2017.2694410

12 C. Yang, C. Chen, N. Wang, Z. Ju, J. Fu, and M. Wang: IEEE Trans. Cognit. Dev. Syst. 11 (2019) 281. https:// doi: 10.1109/TCDS.2018.2866477

13 X. Jie, L. Liu, and R. Zhang: IEEE Trans. Signal Process. 62 (2014) 4798. https://doi.org/10.1109/ TSP.2014.2340817

14 J. Xu and R. Zhang: IEEE Trans. Signal Process. 62 (2014) 5370. https://doi.org/10.1109/TSP.2014.2352604

15 Y. Zeng and R. Zhang: IEEE Trans. Commun. 63 (2015) 536. https://doi.org/10.1109/TCOMM.2014.2385077

16 W. Fei, S. H. Shao, and Y. X. Tang: Acta Electr. 45 (2017) 372. https://doi.org/10.3969/j.issn.0372-2112.2017.01.002

17 B. Clerckx and E. Bayguzina: IEEE Trans. Signal Process. 64 (2016) 6313. https://doi.org/10.1109/ TSP.2016.2601284

18 J. Xu and R. Zhang: IEEE Trans. Signal Process. 64 (2016) 2475. https://doi.org/10.1109/TSP.2016.2526965

19 A. Kurs, R. Moffatt, and S. Marin: Appl. Phys. Lett. 96 (2010) 2475. https://doi.org/10.1063/1.3284651

20 G. Rishi and C. Jorge: J. Int. Rob. 22 (2015) 504. https://doi.org/10.1002/rnc.1710

21 C. Yang, G. Peng, Y. Li, R. Cui, L. Cheng, and Z. Li: IEEE Trans. Cybern. 49 (2018) 2568. https://doi. org/10.1109/TCYB.2018.2828654

22 G. Cena, S. Scanzio, L. Seno, and A. Valenzano: 11th IEEE Symp. Industrial Embedded Systems (SIES) 34 (2016) 1. https://doi.org/10.1109/SIES.2016.7509416

23 Z. Yong, R. Zhang, and T. J. Lim: IEEE Commun. Mag. 54 (2016) 36. https://doi.org/10.1109/ MCOM.2016.7470933

24 Y. Li, R. Cui, and Z. Li: IEEE Trans. Ind. Electron. 65 (2018) 8718. https://doi.org/10.1109/TIE.2018.2816000

25 H. Xiao, R. Cui, and D. Xu: IEEE Trans. Cybern. 48 (2017) 1773. https://doi.org/10.1109/TCYB.2017.2715228

26 L. Chen, R. Cui, C. Yang, and W. Yan: IEEE Trans. Ind. Electron. 14 (2019) 568. https://doi.org/10.1 109/ TIE.2019.2914631

27 Z. Yong, R. Zhang, and T. J. Lim: IEEE Trans. Commun. 64 (2016) 4983. https://doi.org/10.1109/ TCOMM.2016.2611512

28 W. B. Heinzelman, A. P. Chandrakasan, and H. Balakrishnan: IEEE Trans. Wireless Commun. 1 (2016) 660. https://doi.org/10.1109/TCOMM.2016.2611512

29 J. Li and G. AlRegib: IEEE Mil. Commun. Conf. 5 (2006) 7. https://doi.org/10.1109/MILCOM.2006.302486

30 H. Huang. T. Zhang, C. Yang, and C. L. P. Chen: IEEE Trans. Ind. Electron. 29 (2019) 1. https://doi. org/10.1109/TIE.2019.2950853

\section{About the Authors}

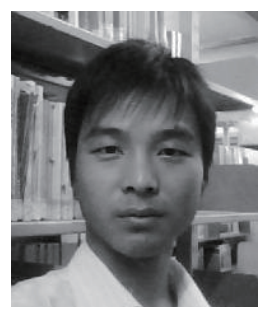

Guiyun Liu received his B.Sc. degree from Qinghai University, Xining, China, in 2006, and his Ph.D. degree from South China University of Technology, Guangzhou, China, in 2012. After that, he joined the School of Mechanical and Electric Engineering, Guangzhou University, China, where he is now an associate professor. His research interests are in energy-efficient wireless communications.

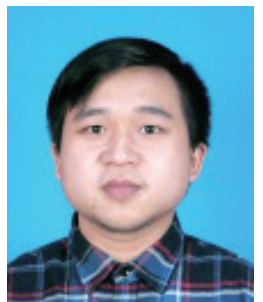

Wenjun Jiang received his B.Sc. degree from Hunan University of Arts and Science, Changde, China, in 2013. He is a graduate student of electronic and communication engineering in the College of Mechanical and Electrical Engineering, Guangzhou University. His research interests are in wireless power transfer and energy-efficient wireless communications. 


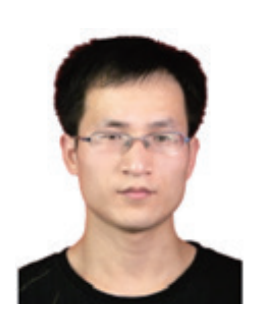

Weiqiang Tan received his M.S. degree from Chengdu University of Information Technology, China, in 2013, and his Ph.D. degree from the National Mobile Communications Research Laboratory, Southeast University, Nanjing, China, in 2017. From 2016 to 2017, he was a visiting Ph.D. student at the School of Electronics, Electrical Engineering and Computer Science, Queen's University Belfast, United Kingdom. He is currently with the School of Computer Science and Cyber Engineering, Guangzhou University, Guangzhou, China. His research interests are in massive MIMO systems and millimeter wave wireless communication.

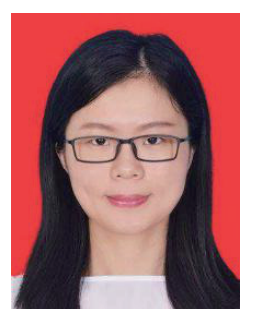

Xuejing Lan received her Ph.D. degree in control science and engineering from Huazhong University of Science and Technology, Wuhan, China, in 2017. She is currently a lecturer at the School of Mechanical and Electrical Engineering, Guangzhou University, Guangzhou, China. Her research interests are in multi-agent systems and trajectory planning control. 